\title{
A Comparison of Distributed Spatial Data Management Systems for Processing Distance Join Queries
}

\author{
Francisco García-García ${ }^{1, \star}$, Antonio Corral ${ }^{1, \star}$, Luis Iribarne ${ }^{1, \star}$, \\ George Mavrommatis ${ }^{2}$, and Michael Vassilakopoulos ${ }^{2, \star}$ \\ 1 Dept. of Informatics, University of Almeria, Almeria, Spain. \\ E-mail: \{paco.garcia, acorral, liribarn\}@ual.es \\ 2 DaSE Lab, Dept. of Electrical and Computer Eng., University of Thessaly, \\ Volos, Greece. E-mail: gmav, mvasilako@uth.gr
}

\begin{abstract}
Due to the ubiquitous use of spatial data applications and the large amounts of spatial data that these applications generate, the processing of large-scale distance joins in distributed systems is becoming increasingly popular. Two of the most studied distance join queries are the $K$ Closest Pair Query (KCPQ) and the $\varepsilon$ Distance Join Query $(\varepsilon D J Q)$. The $K C P Q$ finds the $K$ closest pairs of points from two datasets and the $\varepsilon D J Q$ finds all the possible pairs of points from two datasets, that are within a distance threshold $\varepsilon$ of each other. Distributed clusterbased computing systems can be classified in Hadoop-based and Sparkbased systems. Based on this classification, in this paper, we compare two of the most current and leading distributed spatial data management systems, namely SpatialHadoop and LocationSpark, by evaluating the performance of existing and newly proposed parallel and distributed distance join query algorithms in different situations with big real-world datasets. As a general conclusion, while SpatialHadoop is more mature and robust system, LocationSpark is the winner with respect to the total execution time.
\end{abstract}

Keywords: Spatial Data Processing, Distance Joins, SpatialHadoop, LocationSpark.

\section{Introduction}

Nowadays, the volume of available spatial data (e.g. location, routing, navigation, etc.) is increasing hugely across the world-wide. Recent developments of spatial big data systems have motivated the emergence of novel technologies for processing large-scale spatial data on clusters of computers in a distributed fashion. These Distributed Spatial Data Management Systems (DSDMSs) can be classified in disk-based [9] and in-memory [19] ones. The disk-based DSDMSs are characterized by being Hadoop-based systems and the most representative ones

* Work funded by the MINECO research project [TIN2013-41576-R] 
are Hadoop-GIS [1] and SpatialHadoop [6]. The Hadoop-based systems enable to execute spatial queries using predefined high-level spatial operators without having to worry about fault tolerance and computation distribution. On the other hand, the in-memory DSDMSs are characterized as Spark-based systems and the most representative ones are SpatialSpark [15], GeoSpark [17], Simba [14] and LocationSpark [12,13]. The Spark-based systems allow users to work on distributed in-memory data without worrying about the data distribution mechanism and fault-tolerance.

Distance join queries (DJQs) have received considerable attention from the database community, due to their importance in numerous applications, such as spatial databases and GIS, data mining, multimedia databases, etc. DJQs are costly queries because they combine two datasets taking into account a distance metric. Two of the most representative ones are the $K$ Closest Pair Query $(K \mathrm{CPQ})$ and the $\varepsilon$ Distance Join Query $(\varepsilon D J Q)$. Given two point datasets $\mathbb{P}$ and $\mathbb{Q}$, the $K \mathrm{CPQ}$ finds the $K$ closest pairs of points from $\mathbb{P} \times \mathbb{Q}$ according to a certain distance function (e.g., Manhattan, Euclidean, Chebyshev, etc.). The $\varepsilon D J Q$ finds all the possible pairs of points from $\mathbb{P} \times \mathbb{Q}$, that are within a distance threshold $\varepsilon$ of each other. Several research works have been devoted to improve the performance of these queries by proposing efficient algorithms in centralized environments $[2,10]$. But, with the fast increase in the scale of the big input datasets, processing large data in parallel and distributed fashions is becoming a popular practice. For this reason, a number of parallel algorithms for DJQs in MapReduce [3] and Spark [18] have been designed and implemented [7,13].

Apache Hadoop ${ }^{1}$ is a reliable, scalable, and efficient cloud computing framework allowing for distributed processing of large datasets using MapReduce programming model. However, it is a kind of disk-based computing framework, which writes all intermediate data to disk between map and reduce tasks. MapReduce [3] is a framework for processing and managing large-scale datasets in a distributed cluster. It was introduced with the goal of providing a simple yet powerful parallel and distributed computing paradigm, providing good scalability and fault tolerance mechanisms. Apache Spark ${ }^{2}$ is a fast, reliable and distributed in-memory large-scale data processing framework. It takes advantage of the Resilient Distributed Dataset (RDD), which allows transparently storing data in memory and persisting it to disk only if it is needed [18]. Hence, it can reduce a huge number of disk writes and reads to outperform the Hadoop platform. Since Spark maintains the status of assigned resources until a job is completed, it reduces time consumption in resource preparation and collection.

Both Hadoop and Spark have weaknesses related to efficiency when applied to spatial data. A main shortcoming is the lack of any indexing mechanism that would allow selective access to specific regions of spatial data, which would in turn yield more efficient query processing algorithms. A solution to this problem is an extension of Hadoop, called SpatialHadoop [6], which is a framework that supports spatial indexing on top of Hadoop, i.e. it adopts two-level index struc-

\footnotetext{
1 Available at https://hadoop.apache.org/

2 Available at https://spark.apache.org/
} 
ture (global and local) to organize the stored spatial data. And other possible solution is LocationSpark [12,13], which is a spatial data processing system built on top of Spark and it employs various spatial indexes for in-memory data.

In the literature, up to now, there are only few comparative studies between Hadoop-based and Spark-based systems. The most representative one is [11], for a general perspective. But, for comparing DSDMSs, we can find $[16,17,8]$. Motivated by this fact, in this paper we compare SpatialHadoop and LocationSpark for distance-based join query processing, in particular for KCPQ and $\varepsilon \mathrm{DJQ}$, in order to provide criteria for adopting one or the other DSDMS. The contributions of this paper are the following:

- Novel algorithms in LocationSpark (the first ones in the literature) to perform efficient parallel and distributed $K \mathrm{CPQ}$ and $\varepsilon \mathrm{DJQ}$, on big real-world spatial datasets

- The execution of a set of experiments for comparing the performance of the two DSDMSs (SpatialHadoop and LocationSpark).

- The execution of a set of experiments for examining the efficiency and the scalability of the existing and new DJQ algorithms.

This paper is organized as follows. In Section 2, we review related work on Hadoop-based and Spark-based systems that support spatial operations and provide the motivation for this paper. In Section 3, we present preliminary concepts related to DJQs, SpatialHadoop and LocationSpark. In Section 4, the parallel algorithms for processing $K \mathrm{CPQ}$ and $\varepsilon \mathrm{DJQ}$ in LocationSpark are proposed. In Section 5, we present representative results of the extensive experimentation that we have performed, using real-world datasets, for comparing these two cloud computing frameworks. Finally, in Section 6, we provide the conclusions arising from our work and discuss related future work directions.

\section{Related Work and Motivation}

Researchers, developers and practitioners worldwide have started to take advantage of the cluster-based systems to support large-scale data processing. There exist several cluster-based systems that support spatial queries over distributed spatial datasets and they can be classified in Hadoop-based and Spark-based systems. The most important contributions in the context of Hadoop-based systems are the following research prototypes:

- Hadoop-GIS [1] extends Hive and adopts Hadoop Streaming framework and integrates several open source software packages for spatial indexing and geometry computation. Hadoop-GIS only supports data up to two dimensions and two query types: rectangle range query and spatial joins.

- SpatialHadoop [6] is an extension of the MapReduce framework [3], based on Hadoop, with native support for spatial $2 \mathrm{~d}$ data (see section 3.2 ).

On the other hand, the most remarkable contributions in the context of Spark-based systems are the following prototypes: 
- SpatialSpark [15] is a lightweight implementation of several spatial operations on top of the Spark in-memory big data system. It targets at in-memory processing for higher performance. SpatialSpark adopts data partition strategies like fixed grid or kd-tree on data files in HDFS and builds an index to accelerate spatial operations. It supports range queries and spatial joins over geometric objects using conditions like intersect and within.

- GeoSpark [17] extends Spark for processing spatial data. It provides a new abstraction called Spatial Resilient Distributed Datasets (SRDDs) and a few spatial operations. It allows an index (e.g. Quadtree and R-tree) to be the object inside each local RDD partition. For the query processing point of view, GeoSpark supports range query, $K \mathrm{NNQ}$ and spatial joins over SRDDs.

- Simba (Spatial In-Memory Big data Analytics) [14] offers scalable and efficient in-memory spatial query processing and analytics for big spatial data. Simba is based on Spark and runs over a cluster of commodity machines. In particular, Simba extends the Spark SQL engine to support rich spatial queries and analytics through both SQL and the DataFrame API. It introduces partitioning techniques (e.g. STR), indexes (global and local) based on R-trees over RDDs in order to work with big spatial data and complex spatial operations (e.g. range query, $K \mathrm{NNQ}$, distance join and $K \mathrm{NNJQ}$ ).

- LocationSpark $[12,13]$ is an efficient in-memory distributed spatial query processing system (see section 3.3 for more details).

As we have seen, there are several distributed systems based on Hadoop or Spark for managing spatial data, but there are not many articles comparing them with respect to spatial query processing. The only contributions in this regard are $[16,17,8]$. In $[16,17]$, SpatialHadoop is compared with SpatialSpark and GeoSpark, respectively, for spatial join query processing. In [8], SpatialHadoop is compared with GeoSpark with respect to the architectural point of view. Motivated by these observations, and since $K \mathrm{CPQ}$ [7] is implemented in SpatialHadoop (its adaptation to $\varepsilon$ DJQ is straightforward), and in LocationSpark neither $K \mathrm{CPQ}$ nor $\varepsilon \mathrm{DJQ}$ have been implemented yet, we design and implement both DJQs in LocationSpark. Moreover, we develop a comparative performance study between SpatialHadoop and LocationSpark for KCPQ and $\varepsilon \mathrm{DJQ}$.

\section{Preliminaries and Background}

In this section, we first present the basic definitions of the $K \mathrm{CPQ}$ and $\varepsilon \mathrm{DJQ}$, followed by a brief introduction of the preliminary concepts about SpatialHadoop and LocationSpark, the DSDMSs to be compared.

\subsection{The $K$ Closest Pairs and $\varepsilon$ Distance Join Queries}

The $K \mathrm{CPQ}$ discovers the $K$ pairs of data formed from the elements of two datasets having the $K$ smallest distances between them (i.e. it reports only the top $K$ pairs). The formal definition of the $K \mathrm{CPQ}$ for point datasets (the 
extension of this definition to other, more complex spatial objects - e.g. linesegments, objects with extents, etc. - is straightforward) is the following:

Definition 1. ( $K$ Closest Pairs Query, $K$ CPQ)

Let $\mathbb{P}=\left\{p_{0}, p_{1}, \cdots, p_{n-1}\right\}$ and $\mathbb{Q}=\left\{q_{0}, q_{1}, \cdots, q_{m-1}\right\}$ be two set of points, and a number $K \in \mathbb{N}^{+}$. Then, the result of the $K$ Closest Pairs Query is an ordered collection, $K C P Q(\mathbb{P}, \mathbb{Q}, K)$, containing $K$ different pairs of points from $\mathbb{P} \times \mathbb{Q}$, ordered by distance, with the $K$ smallest distances between all possible pairs:

$K C P Q(\mathbb{P}, \mathbb{Q}, K)=\left(\left(p_{1}, q_{1}\right),\left(p_{2}, q_{2}\right), \cdots,\left(p_{K}, q_{K}\right)\right),\left(p_{i}, q_{i}\right) \in \mathbb{P} \times \mathbb{Q}, 1 \leq i \leq K$, such that for any $(p, q) \in \mathbb{P} \times \mathbb{Q} \backslash K C P Q(\mathbb{P}, \mathbb{Q}, K)$ we have $\operatorname{dist}\left(p_{1}, q_{1}\right) \leq$ $\operatorname{dist}\left(p_{2}, q_{2}\right) \leq \cdots \leq \operatorname{dist}\left(p_{K}, q_{K}\right) \leq \operatorname{dist}(p, q)$.

Note that if multiple pairs of points have the same $K$-th distance value, more than one collection of $K$ different pairs of points are suitable as a result of the query. Recall that $K \mathrm{CPQ}$ is implemented in SpatialHadoop [7] using plane-sweep algorithms [10], but not in LocationSpark.

On the other hand, the $\varepsilon$ DJQ reports all the possible pairs of spatial objects from two different spatial objects datasets, $\mathbb{P}$ and $\mathbb{Q}$, having a distance smaller than a distance threshold $\varepsilon$ of each other [10]. The formal definition of $\varepsilon$ DJQ for point datasets is the following:

Definition 2. ( $\varepsilon$ Distance Join Query, $\varepsilon$ DJQ)

Let $\mathbb{P}=\left\{p_{0}, p_{1}, \cdots, p_{n-1}\right\}$ and $\mathbb{Q}=\left\{q_{0}, q_{1}, \cdots, q_{m-1}\right\}$ be two set of points, and a distance threshold $\varepsilon \in \mathbb{R}_{\geq 0}$. Then, the result of the $\varepsilon D J Q$ is the set, $\varepsilon D J Q(\mathbb{P}, \mathbb{Q}, \varepsilon) \subseteq \mathbb{P} \times \mathbb{Q}$, containing all the possible different pairs of points from $\mathbb{P} \times \mathbb{Q}$ that have a distance of each other smaller than, or equal to $\varepsilon$ :

$\varepsilon D J Q(\mathbb{P}, \mathbb{Q}, \varepsilon)=\left\{\left(p_{i}, q_{j}\right) \in P \times Q: \operatorname{dist}\left(p_{i}, q_{j}\right) \leq \varepsilon\right\}$

The $\varepsilon \mathrm{DJQ}$ can be considered as an extension of the $K \mathrm{CPQ}$, where the distance threshold of the pairs is known beforehand and the processing strategy (e.g. plane-sweep technique) can be the same as in the KCPQ for generating the candidate pairs of the final result. For this reason, its adaptation to SpatialHadoop from $K \mathrm{CPQ}$ is straightforward. Note that $\varepsilon \mathrm{DJQ}$ is not implemented in LocationSpark.

\section{$3.2 \quad$ SpatialHadoop}

SpatialHadoop [6] is a full-fledged MapReduce framework with native support for spatial data. It is an efficient disk-based distributed spatial query processing system. Note that MapReduce [3] is a scalable, flexible and fault-tolerant programming framework for distributed large-scale data analysis. A task to be performed using the MapReduce framework has to be specified as two phases: the map phase is specified by a map function takes input (typically from Hadoop Distributed File System (HDFS) files), possibly performs some computations on this input, and distributes it to worker nodes; and the reduce phase which processes these results as specified by a reduce function. Additionally, a combiner 
function can be used to run on the output of map phase and perform some filtering or aggregation to reduce the number of keys passed to the reducer.

SpatialHadoop is a comprehensive extension to Hadoop that injects spatial data awareness in each Hadoop layer, namely, the language, storage, MapReduce, and operations layers. MapReduce layer is the query processing layer that runs MapReduce programs, taking into account that SpatialHadoop supports spatially indexed input files. The Operation layer enables the efficient implementation of spatial operations, considering the combination of the spatial indexing in the storage layer with the new spatial functionality in the MapReduce layer. In general, a spatial query processing in SpatialHadoop consists of four steps $[6,7]$ (see Figure 1): (1) Preprocessing, where the data is partitioned according to a specific spatial index, generating a set of partitions or cells. (2) Pruning, when the query is issued, where the master node examines all partitions and prunes by a filter function those ones that are guaranteed not to include any possible result of the spatial query. (3) Local Spatial Query Processing, where a local spatial query processing is performed on each non-pruned partition in parallel on different machines (map tasks). Finally, (4) Global Processing, where the results are collected from all machines in the previous step and the final result of the concerned spatial query is computed. A combine function can be applied in order to decrease the volume of data that is sent from the map task. The reduce function can be omitted when the results from the map phase are final.

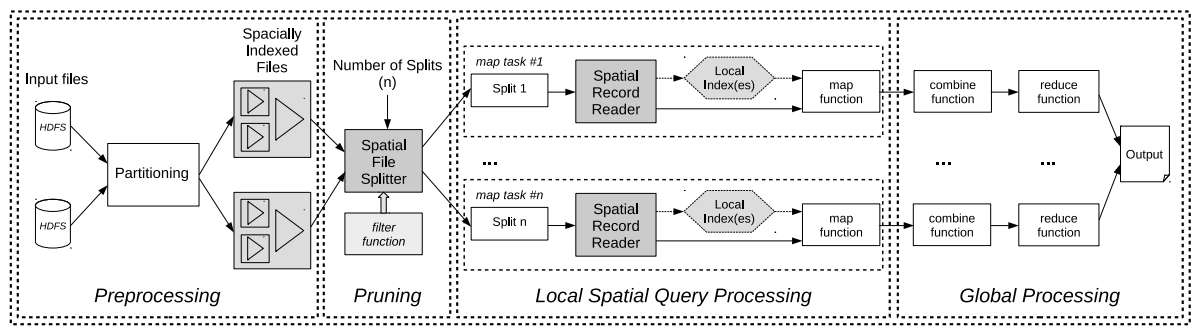

Fig. 1. Spatial query processing in SpatialHadoop [6, 7].

\subsection{LocationSpark}

LocationSpark $[12,13]$ is a library in Spark that provides an API for spatial query processing and optimization based on Spark's standard dataflow operators. It is an efficient in-memory distributed spatial query processing system. LocationSpark provides several optimizations to enhance Spark for managing spatial data and they are organized by layers: memory management, spatial index, query executor, query scheduler, spatial operators and spatial analytical. In the Memory Management layer for spatial data, LocationSpark dynamically caches frequently accessed data into memory, and stores the less frequently used data into disk. For the Spatial Index layer, LocationSpark builds two levels of 
spatial indexes (global and local). To build a global index, LocationSpark samples the underlying data to learn the data distribution in space and provides a grid and a region Quadtree. In addition, each data partition has a local index (e.g., a grid local index, an R-tree, a variant of the Quadtree, or an IR-tree). Finally, LocationSpark adopts a new Spatial Bloom Filter to reduce the communication cost when dispatching queries to their overlapping data partitions, termed sFilter, that can speed up query processing by avoiding needless communication with data partitions that do not contribute to the query answer. In the Query Executor layer, LocationSpark evaluates the runtime and memory usage trade-offs for the various alternatives, and then, it chooses and executes the better execution plan on each slave node. LocationSpark has a new layer, termed Query Scheduler, with an automatic skew analyzer and a plan optimizer to mitigate query skew. The query scheduler uses a cost model to analyze the skew to be used by the spatial operators, and a plan generation algorithm to construct a load-balanced query execution plan. After plan generation, local computation nodes select the proper algorithms to improve their local performance based on the available spatial indexes and the registered queries on each node. For the Spatial Operators layer, LocationSpark supports spatial querying and spatial data updates. It provides a rich set of spatial queries including spatial range query, $K N N Q$, spatial-join, and $K$ NNJQ. Moreover, it supports data updates and spatio-textual operations. Finally, for the Spatial Analytical layer, and due to the importance of spatial data analysis, LocationSpark provides spatial data analysis functions including spatial data clustering, spatial data skyline computation and spatio-textual topic summarization. Since our main objective is to include the DJQs ( $K \mathrm{CPQ}$ and $\varepsilon \mathrm{DJQ}$ ) into LocationSpark, we are interested in the Spatial Operators layer, where we will implement them.

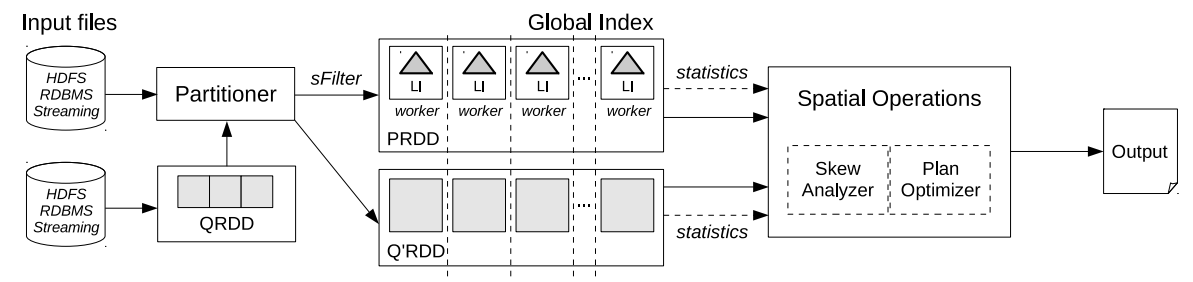

Fig. 2. Spatial query processing for DJQs in LocationSpark, based on [13].

To process spatial queries, LocationSpark builds a distributed spatial index structure for in-memory spatial data. As we can see in Figure 2, for DJQs, given two datasets $\mathbb{P}$ and $\mathbb{Q}, \mathbb{P}$ is partitioned into $N$ partitions based on a spatial index criteria (e.g. $N$ leaves of a R-tree) by the Partitioner leading to the PRDD (Global Index). The sFilter determines whether a point is contained inside a spatial range or not. Next, each worker has a local data partition $\mathbb{P}_{i}(1 \leq i \leq$ $N$ ) and builds a Local Index (LI). $Q R D D$ is generated from $\mathbb{Q}$ by a member function of RDD (Resilient Distributed Dataset) natively supported by Spark, 
that forwards such point to the partitions that spatially overlap it. Now, each point of $\mathbb{Q}$ is replicated to the partitions that are identified using the $P R D D$ (Global Index), leading to the $Q^{\prime} R D D$. Then a post-processing step (using the Skew Analyzer and the Plan Optimizer) is performed to combine the local results to generate the final output.

\section{DJQ Algorithms in SpatialHadoop and LocationSpark}

Since $K C P Q$ is already implemented in SpatialHadoop [7], in this section, we will present how we can adapt $K \mathrm{CPQ}$ to $\varepsilon \mathrm{DJQ}$ in SpatialHadoop and how $K \mathrm{CPQ}$ and $\varepsilon \mathrm{DJQ}$ can be implemented in LocationSpark.

\subsection{KCPQ and $\varepsilon \mathrm{DJQ}$ in SpatialHadoop}

In general, the $K \mathrm{CPQ}$ algorithm in SpatialHadoop [7] consists of a MapReduce job. The map function aims to find the $K \mathrm{CP}$ between each local pair of partitions from $\mathbb{P}$ and $\mathbb{Q}$ with a particular plane-sweep $K \mathrm{CPQ}$ algorithm [10] and the result is stored in a binary max heap (called LocalKMaxHeap). The reduce function aims to examine the candidate pairs of points from each LocalKMaxHeap and return the final set of the $K$ closest pairs in another binary max heap (called GlobalKMaxHeap). To improve this approach, for reducing the number of possible combinations of pairs of partitions, we need to find in advance an upper bound of the distance value of the $K$-th closest pair of the joined datasets, called $\beta$. This $\beta$ computation can be carried out by sampling globally from both datasets or by sampling locally for an appropriate pair of partitions and, then executing a plane-sweep $K \mathrm{CPQ}$ algorithm over both samples.

The method for the $\varepsilon D J Q$ in MapReduce, adapting from $K C P Q$ in SpatialHadoop [7], adopts the map phase of the join MapReduce methodology. The idea is to have $\mathbb{P}$ and $\mathbb{Q}$ partitioned by some method (e.g., Grid) into two sets of cells, with $n$ and $m$ cells of points, respectively. Then, every possible pair of cells is sent as input for the filter function. This function takes as input, combinations of pairs of cells in which the input set of points are partitioned and a distance threshold $\varepsilon$, and it prunes pairs of cells which have minimum distances larger than $\varepsilon$. By using SpatialHadoop built-in function MinDistance we can calculate the minimum distance between two cells (i.e. this function computes the minimum distance between the two MBRs, Minimum Bounding Rectangles, of the two cells). On the map phase, each mapper reads the points of a pair of filtered cells and performs a plane-sweep $\varepsilon \mathrm{DJQ}$ algorithm [10] (variation of the plane-sweep-based $K \mathrm{CPQ}$ algorithm) between the points inside that pair of cells. The results from all mappers are just combined in the reduce phase and written into HDFS files, storing only the pairs of points with distance up to $\varepsilon$.

\subsection{K KPQ and $\varepsilon \mathrm{DJQ}$ in LocationSpark}

Assuming that $\mathbb{P}$ is the largest dataset to be combined and $\mathbb{Q}$ is the smallest one, and following the ideas presented in [13], we can describe the Execution 
Plan for KCPQ in LocationSpark as follows. In Stage 1, the two datasets are partitioned according to a given spatial index schema. In Stage 2, statistic data is added to each partition, $S_{\mathbb{P}}$ and $S_{\mathbb{Q}}$, and they are combined by pairs, $S_{\mathbb{P Q}}$. In Stage 3, the partitions from $\mathbb{P}$ and $\mathbb{Q}$ with the largest density of points, $\mathbb{P}_{\beta}$ and $\mathbb{Q}_{\beta}$, are selected to be combined by using a plane-sweep $K \mathrm{CPQ}$ algorithm [10] to compute an upper bound of the distance value of the $K$-th closest pair $(\beta)$. In Stage 4 , the combination of all possible pairs of partitions from $\mathbb{P}$ and $\mathbb{Q}, S_{\mathbb{P Q}}$, is filtered according to the $\beta$ value (i.e. only the pairs of partitions with minimum distance between the MBRs of the partitions is smaller than or equal to $\beta$ are selected), giving rise to $F S_{\mathbb{P Q}}$, and all pairs of filtered partitions are processed by using a plane-sweep $K \mathrm{CPQ}$ algorithm. Finally, the results are merged to get the final output.

With the previous Execution Plan and increasing the size of the datasets, the execution time increases considerably due to skew and shuffle problems. To solve it, we modify Stage 4 with the query plan that is used for the algorithms shown in [13], leaving the plan as shown in Figure 3.

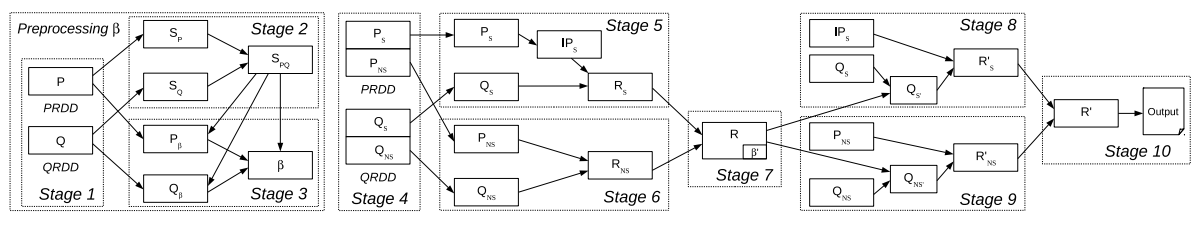

Fig. 3. Execution Plan for $K \mathrm{CPQ}$ in LocationSpark, based on [13].

Stages 1, 2 and 3 are still used to calculate the $\beta$ value which will serve to accelerate the local pruning phase on each partition. In Stage 4, using the Query Plan Scheduler, $\mathbb{P}$ is partitioned into $\mathbb{P}_{\mathbb{S}}$ and $\mathbb{P}_{\mathbb{N S}}$ being the partitions that present and do not present skew, respectively. The same partitioning is used to $\mathbb{Q}$. In Stage 5, a $K \mathrm{CPQ}$ algorithm [10] is applied between points of $\mathbb{P}_{\mathbb{S}}$ and $\mathbb{Q}_{\mathbb{S}}$ that are in the same partition and likewise for $\mathbb{P}_{\mathbb{N S}}$ and $\mathbb{Q}_{\mathbb{N S}}$ in Stage 6. These two stages are executed independently and the results are combined in Stage 7. Finally, it is still necessary to calculate if there is any present candidate for each partition that is on the boundaries of that same partition in the other dataset. To do this, we use $\beta^{\prime}$ which is the maximum distance from the current set of candidates as a radius of a range filter with center in each partition to obtain possible new candidates on those boundaries. The calculation of $K \mathrm{CPQ}$ of each partition with its candidates is executed in Stages 8 and 9 and these results are combined in Stage 10 to obtain the final answer.

The Execution Plan for $\varepsilon \mathrm{DJQ}$ in LocationSpark is a variation of the $K \mathrm{CPQ}$ one, where the filtering stages are removed, since $S_{\mathbb{P Q}}$ is filtered by $\varepsilon$ (i.e. $\beta=$ $\beta^{\prime}=\varepsilon$ ), which is the threshold distance known beforehand. 


\section{Experimentation}

In this section we present the results of our experimental evaluation. We have used real 2d point datasets to test our DJQ algorithms in SpatialHadoop and LocationSpark. We have used three datasets from OpenStreetMap ${ }^{3}$ : BUILDINGS which contains $115 \mathrm{M}$ records of buildings, LAKES which contains $8.4 \mathrm{M}$ points of water areas, and PARKS which contains $10 \mathrm{M}$ records of parks and green areas [6]. Moreover, to experiment with the biggest real dataset (BUILDINGS), we have created a new big quasi-real dataset from $L A K E S(8.4 \mathrm{M})$, with a similar quantity of points. The creation process is as follows: taking one point of $L A$ $K E S$, $p$, we generate 15 new points gathered around $p$ (i.e. the center of the cluster), according to a Gaussian distribution with mean $=0.0$ and standard deviation $=0.2$, resulting in a new quasi-real dataset, called CLUS_LAKES, with around $126 \mathrm{M}$ of points. The main performance measure that we have used in our experiments has been the total execution time (i.e. total response time). All experiments are conducted on a cluster of 12 nodes on an OpenStack environment. Each node has $4 \mathrm{vCPU}$ with $8 \mathrm{~GB}$ of main memory running Linux operating systems and Hadoop 2.7.1.2.3. Each node has a capacity of 3 vCores for MapReduce2 / YARN use. The version of Spark used is 1.6.2. Finally, we used the latest code available in the repositories of SpatialHadoop ${ }^{4}$ and LocationSpark ${ }^{5}$.

\begin{tabular}{|c|c|}
\hline Parameter & Values (default) \\
\hline \hline$K$ & $1,10,\left(10^{2}\right), 10^{3}, 10^{4}, 10^{5}$ \\
\hline$\varepsilon\left(\times 10^{-4}\right)$ & $2.5,5,7.5,12.5,(25), 50$ \\
\hline Number of nodes & $1,2,4,6,8,10,(12)$ \\
\hline Type of partition & Quadtree \\
\hline
\end{tabular}

Table 1. Configuration parameters used in the experiments.

Table 1 summarizes the configuration parameters used in our experiments. Default values (in parentheses) are used unless otherwise mentioned. SpatialHadoop needs the datasets to be partitioned and indexed before invoking the spatial operations. The times needed for that pre-processing phase are 94 secs for $L A K E S, 103 \mathrm{sec}$ for PARKS, $175 \mathrm{sec}$ for BUILDINGS and $200 \mathrm{sec}$ for CLUS_LAKES. We have shown the time of this pre-processing phase in SpatialHadoop (disk-based DSDMS), since it would be the full execution time, at least in the first running of the query. Note that, data are indexed and the index is stored on HDFS and for subsequent spatial queries, data and index are already available (this can be considered as an advantage of SpatialHadoop). On the other hand, LocationSpark (in-memory-based DSDMS) always partitions and indexes the data for every operation. The partitions/indexes are not stored on any persistent file system and cannot be reused in subsequent operations.

\footnotetext{
3 Available at http://spatialhadoop.cs.umn.edu/datasets.html

4 Available at https://github.com/aseldawy/spatialhadoop2

${ }^{5}$ Available at https://github.com/merlintang/SpatialSpark
} 
Our first experiment aims to measure the scalability of the $K \mathrm{CPQ}$ and $\varepsilon \mathrm{DJQ}$ algorithms, varying the dataset sizes. As shown in the left chart of Figure 4 for the $K \mathrm{CPQ}$ of real datasets $(L A K E S \times P A R K S, B U I L D I N G S \times P A R K S$ and BUILDINGS $\times$ CLUS_LAKES), the execution times in both DSDMSs increase linearly as the size of the datasets increase. Moreover, LocationSpark is faster for all the datasets combinations except for the largest one (e.g. it is 29 sec slower for the biggest datasets, BUILDINGS $\times$ CLUS_LAKES (BxC_L)). However, it should be noted that SpatialHadoop needs a pre-indexing time of 175 and $200 \mathrm{sec}$ for each dataset (depicted by vertical lines in the charts) and that difference can be caused by memory constraints on the cluster.

As we have just seen for $K \mathrm{CPQ}$, the behavior of the execution times when varying the size of the datasets is very similar for $\varepsilon \mathrm{DJQ}$. For instance, for the combination of large datasets (see the right chart of Figure 4), BUILDINGS $\times$ CLUS_LAKES (BxC_L), SpatialHadoop is $32 \mathrm{sec}$ faster than LocationSpark. However, for smaller sets, LocationSpark shows better performance (e.g. it is 96 sec faster for the middle size datasets, BUILDINGS $\times$ PARKS $(\mathrm{BxP}))$. From these results with real data, we can conclude that both DSDMSs have similar performance, in terms of execution time, even showing LocationSpark better values in most of the cases, despite the fact that neither pre-partitioning nor pre-indexing are done.

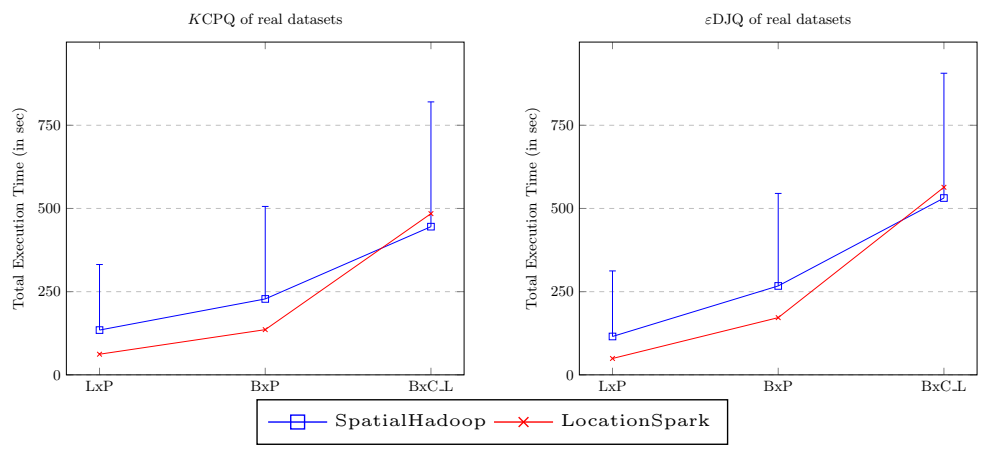

Fig. 4. $K \mathrm{CPQ}$ (left) and $\varepsilon \mathrm{DJQ}$ (right) execution times considering different datasets.

The second experiment studies the effect of the increasing both $K$ and $\varepsilon$ value for the combination of the biggest datasets (BUILDINGS $\times$ CLUS_LAKES). The left chart of Figure 5 shows that the total execution time for real datasets grows slowly as the number of results to be obtained $(K)$ increases. Both DSDMSs, employing Quadtree, report stable execution times, even for large $K$ values (e.g. $K=10^{5}$ ). This means that the Quadtree is less affected by the increment of $K$, because Quadtree employs regular space partitioning depending on the concentration of the points. As shown in the right chart of Figure 5, the total execution time grows as the $\varepsilon$ value increases. Both DSDMSs (SpatialHadoop and LocationSpark) have similar relative performance for all $\varepsilon$ values, with SpatialHadoop being faster, except for $\varepsilon=50 \times 10^{-4}$, where LocationSpark out- 
performs it (i.e. LocationSpark is 377 sec faster). This difference is due to the way in which $\varepsilon$ DJQ is calculated in the latter, where fewer points are used as candidates and skew cells are dealt with its Query Plan Scheduler. For smaller $\varepsilon$ values SpatialHadoop preindexing phase reduces time considerably for very large datasets.

The main conclusions that we can extract for this experiment are: (1) the higher $K$ or $\varepsilon$ values, the greater the possibility that pairs of candidates are not pruned, more tasks would be needed and more total execution time is consumed and, (2) LocationSpark shows better performance especially for higher values of $K$ and $\varepsilon$ thanks to its Query Plan Scheduler and the reduction of the number of candidates.

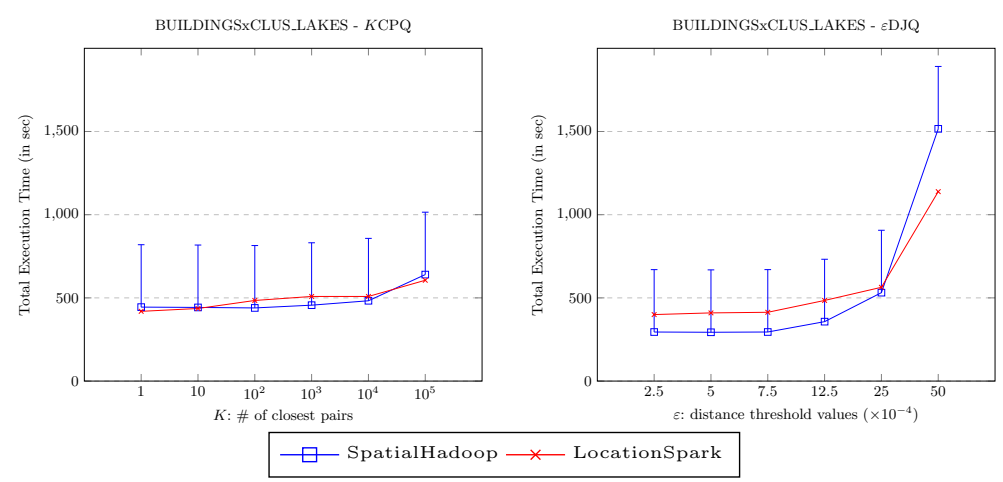

Fig. 5. $K \mathrm{CPQ}$ cost (execution time) vs. $K$ values (left) and $\varepsilon \mathrm{DJQ}$ cost (execution time) vs. $\varepsilon$ values (right).

The third experiment aims to measure the speedup of the DJQ MapReduce algorithms ( $K \mathrm{CPQ}$ and $\varepsilon \mathrm{DJQ}$ ), varying the number of computing nodes $(n)$. The left chart of Figure 6 shows the impact of different number of computing nodes on the performance of parallel $K \mathrm{CPQ}$ algorithm, for BUILDINGS $\times$ PARKS with the default configuration values. From this chart, it could be concluded that the performance of our approach has direct relationship with the number of computing nodes. It could also be deduced that better performance would be obtained if more computing nodes are added. LocationSpark is still showing a better behavior than SpatialHadoop. In the right chart of Figure 6, we can observe a similar trend for $\varepsilon$ DJQ MapReduce algorithm with less execution time, but in this case LocationSpark shows worse performance for a smaller number of nodes. This is due to the fact that LocationSpark and $\varepsilon$ DJQ depends more on the available memory and when the number of nodes decreases, this memory also decreases considerably.

By analyzing the previous experimental results, we can extract several conclusions that are shown below:

- We have experimentally demonstrated the efficiency (in terms of total execution time) and the scalability (in terms of $K$ and $\varepsilon$ values, sizes of datasets 


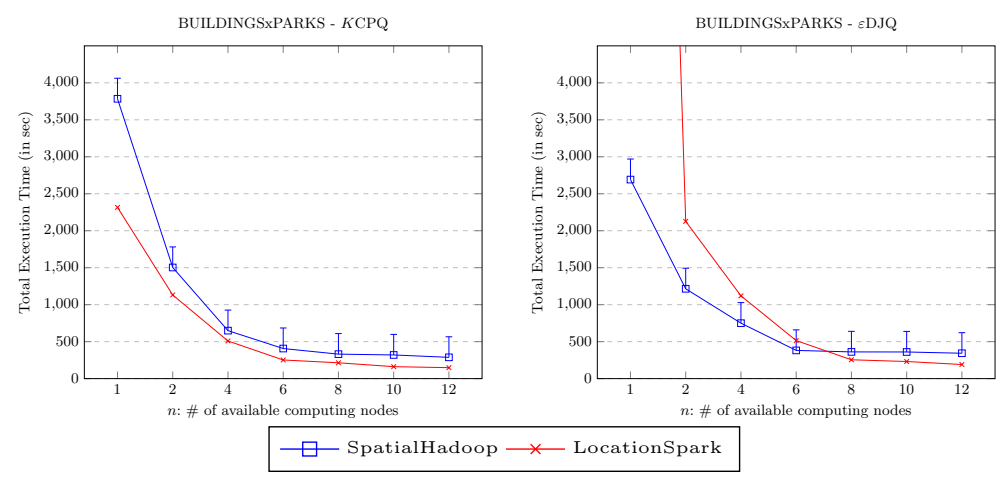

Fig. 6. Query cost with respect to the number of computing nodes $n$.

and number of computing nodes $(n)$ ) of the proposed parallel algorithms for DJQs (KCPQ and $\varepsilon$ DJQ) in SpatialHadoop and LocationSpark.

- The larger the $K$ or $\varepsilon$ values, the larger the probability that pairs of candidates are not pruned, more tasks will be needed and more total execution time is consumed for reporting the final result.

- The larger the number of computing nodes $(n)$, the faster the DJQ algorithms are.

- Both DSDMSs have similar performance, in terms of execution time, although LocationSpark shows better values in most of the cases (if an adequate number of processing nodes with adequate memory resources are provided), despite the fact that neither pre-partitioning nor pre-indexing are done.

\section{Conclusions and Future Work}

The $K \mathrm{CPQ}$ and $\varepsilon \mathrm{DJQ}$ are spatial operations widely adopted by many spatial and GIS applications. These spatial queries have been actively studied in centralized environments, however, for parallel and distributed frameworks has not attracted similar attention. For this reason, in this paper, we compare two of the most current and leading DSDMSs, namely SpatialHadoop and LocationSpark. To do this, we have proposed novel algorithms in LocationSpark, the first ones in literature, to perform efficient parallel and distributed $K \mathrm{CPQ}$ and $\varepsilon \mathrm{DJQ}$ algorithms on big spatial real-world datasets, adopting the plane-sweep technique. The execution of a set of experiments has demonstrated that LocationSpark is the overall winner for the execution time, due to the efficiency of in-memory processing provided by Spark and additional improvements as the Query Plan Scheduler. However, SpatialHadoop is a more mature and robust DSDMS because of time dedicated to investigate and develop it (several years) and, it provides more spatial operations and spatial partitioning techniques. Future work might cover studying other Spark-based DSDMSs like Simba [14], implement other spatial partitioning techniques [4] in LocationSpark and, design and implement other DJQs in these DSDMSs for further comparison. 


\section{References}

1. A. Aji, F. Wang, H. Vo, R. Lee, Q. Liu, X. Zhang and J.H. Saltz: "Hadoop-GIS: A high performance spatial data warehousing system over MapReduce", PVLDB 6(11): 1009-1020, 2013.

2. A. Corral, Y. Manolopoulos, Y. Theodoridis and M. Vassilakopoulos: "Algorithms for processing $K$-closest-pair queries in spatial databases", Data Knowl. Eng. 49(1): 67-104, 2004.

3. J. Dean and S. Ghemawat: "MapReduce: Simplified data processing on large clusters", OSDI Conference, pp. 137-150, 2004.

4. A. Eldawy, L. Alarabi and M.F. Mokbel: "Spatial partitioning techniques in SpatialHadoop", PVLDB 8(12): 1602-1613, 2015.

5. A. Eldawy, Y. Li, M.F. Mokbel and R. Janardan: "CG_Hadoop: computational geometry in MapReduce", SIGSPATIAL Conference, pp. 284-293, 2013.

6. A. Eldawy and M.F. Mokbel: "SpatialHadoop: A MapReduce framework for spatial data", ICDE Conference, pp. 1352-1363, 2015.

7. F. García, A. Corral, L. Iribarne, M. Vassilakopoulos and Y. Manolopoulos: "Enhancing SpatialHadoop with Closest Pair Queries", ADBIS Conference, pp. 212$225,2016$.

8. R.K. Lenka, R.K. Barik, N. Gupta, S.M. Ali, A. Rath and H. Dubey: "Comparative Analysis of SpatialHadoop and GeoSpark for Geospatial Big Data Analytics", CoRR abs/1612.07433, 2016.

9. F. Li, B.C. Ooi, M.T. Özsu and S. Wu: "Distributed data management using MapReduce", ACM Comput. Surv. 46(3): 31:1-31:42, 2014.

10. G. Roumelis, A. Corral, M. Vassilakopoulos and Y. Manolopoulos: "New planesweep algorithms for distance-based join queries in spatial databases", GeoInformatica 20(4): 571-628, 2016.

11. J. Shi, Y. Qiu, U.F. Minhas, L. Jiao, C. Wang, B. Reinwald and F. Özcan: "Clash of the Titans: MapReduce vs. Spark for Large Scale Data Analytics", PVLDB 8(13): 2110-2121, 2015.

12. M. Tang, Y. Yu, Q.M. Malluhi, M. Ouzzani and W.G. Aref: "LocationSpark: A Distributed In-Memory Data Management System for Big Spatial Data", PVLDB 9(13): 1565-1568, 2016.

13. M. Tang, Y. Yu, W.G. Aref, A.R. Mahmood, Q.M. Malluhi and M. Ouzzani: "Inmemory Distributed Spatial Query Processing and Optimization", Available at: http://merlintang.github.io/paper/memory-distributed-spatial.pdf, April, 2017.

14. D. Xie, F. Li, B. Yao, G. Li, L. Zhou and M. Guo: "Simba: Efficient In-Memory Spatial Analytics", SIGMOD Conference, pp. 1071-1085, 2016.

15. S. You, J. Zhang and L. Gruenwald: "Large-scale spatial join query processing in cloud", ICDE Workshops, pp. 34-41, 2015.

16. S. You, J. Zhang and L. Gruenwald: "Spatial join query processing in cloud: Analyzing design choices and performance comparisons", ICPPW Conference, pp. 90-97, 2015.

17. J. Yu, J. Wu and M. Sarwat: "GeoSpark: a cluster computing framework for processing large-scale spatial data", SIGSPATIAL Conference, pp. 70:1-70:4, 2015.

18. M. Zaharia, M. Chowdhury, T. Das, A. Dave, J. Ma, M. McCauly, M.J. Franklin, S. Shenker and I. Stoica: "Resilient Distributed Datasets: A Fault-Tolerant Abstraction for In-Memory Cluster Computing", NSDI Conference, pp. 15-28, 2012.

19. H. Zhang, G. Chen, B.C. Ooi, K.-L. Tan and M. Zhang: "In-Memory Big Data Management and Processing: A Survey", TKDE 27(7): 1920-1948, 2015. 